\title{
A New Test for Multivariate Ordered Alternatives: Medical Application and Geometric Interpretation
}

\author{
Abouzar Bazyari \\ Department of Statistics, Persian Gulf University, Bushehr, Iran \\ ab_bazyari@yahoo.com \\ Mahmoud Afshari \\ Department of Statistics, Persian Gulf University, Bushehr, Iran \\ afshar@pgu.ac.ir
}

\begin{abstract}
In the present paper, the geometric interpretation and medical application of ordered one-sided hypothesis testing are presented. We consider tests for the hypothesis that the mean vectors are zero against one-sided alternatives when the observation vectors are independently and identically distributed as normal with known and unknown covariance matrices. This problem is an extension of Kudo (1963) and Glimm et al. (2002) to several multivariate normal distributions. When the covariance matrices are known, the test statistic is derived and we give suitable conditions which under the one-sided restricted alternative hypothesis the obtained estimator is the maximum likelihood estimate. We shall discuss a geometric interpretation of the test statistic based on the closed convex polyhedral cone and also obtain its null distribution in the special case. Also, when the covariance matrices are unknown and common a proper test statistic is proposed. To evaluate the results, the effect of a drug is investigated on the body of one-day babies for suspected Sepsis disease.
\end{abstract}

Keywords: Geometric interpretation; Medical application; Ordered mean vectors; Polyhedral cone; Sepsis disease.

Mathematics Subject Classification. 62F30; 62H15

\section{Introduction}

Suppose that $\mathbf{X}_{i 1}, \mathbf{X}_{i 2}, \ldots, \mathbf{X}_{i n_{i}}$ are random vectors from a $p$-variate normal distribution with unknown mean vector $\boldsymbol{\mu}_{i}$ and known nonsingular covariance matrix $\boldsymbol{\Sigma}_{i}$, $N_{p}\left(\boldsymbol{\mu}_{i}, \boldsymbol{\Sigma}_{i}\right), i=1,2, \ldots, k$, where we define the space $\mathrm{R}^{p}$ as the parameter space of the mean vectors. We are interested in the problem of testing

$$
\mathrm{H}_{0}: \boldsymbol{\mu}_{1}=\boldsymbol{\mu}_{2}=\cdots=\boldsymbol{\mu}_{k}=\mathbf{0} \text { Versus } \mathrm{H}_{1}: \boldsymbol{\mu}_{1} \geq \mathbf{0}, \boldsymbol{\mu}_{2} \geq \mathbf{0}, \ldots, \boldsymbol{\mu}_{k} \geq \mathbf{0},
$$

where there is at least one strict inequality for components of every vector $\boldsymbol{\mu}_{i}$ in $\mathrm{H}_{1}$ and also $\boldsymbol{\mu}_{i} \geq \mathbf{0}, i=1,2, \ldots, k$, means that all its component are non-negative. The used method to obtain the test statistic for the problem is likelihood ratio criterion. The problem of testing the homogeneity of $k$ univariate normal means against an order restricted alternative hypothesis was discussed in Bartholomew (1959). Much of the development and theory on the ordered subject was assembled by Robertson et al. (1988) and Silvapulle and Sen (2005). Kudo (1963) considered a $p$-dimensional normal distribution with unknown mean $\boldsymbol{\mu}$ and known covariance matrix $\boldsymbol{\Sigma}$. The problem of testing was $\mathrm{H}_{0}^{*}: \boldsymbol{\mu}=\mathbf{0}$ against the restricted alternative $\mathrm{H}_{1}^{*}: \mu_{v} \geq \mathbf{0}, \quad(v=1,2, \ldots, p)$, where the inequality is strict for at least one value of $v$. He obtained a statistic based on 
the likelihood ratio criterion and discussed its existence and gave a scheme for its computation. Perlman (1969) studied this problem under assumption that $\boldsymbol{\Sigma}$ is completely unknown. Sasabuchi et al. (1983) extended Bartholomew's (1959) problem to multivariate normal mean vectors with known covariance matrices and computed the likelihood ratio test.

Glimm et al. (2002) considered tests for the hypothesis that the mean vector is zero against one-sided alternatives when the observation vectors are independently and identically distributed as normal with unknown covariance matrix. Sim and Johnson (2004), considered testing the equality of vectors means against a multivariate ordered alternative under the assumption that the covariance matrix is known and computed the asymptotic distribution of test statistics. Sasabuchi et al. (2003) considered the problem of testing for the case of the covariance matrices are common and unknown. He proposed a test statistic, studied its upper tail probability under the null hypothesis and estimated its critical values. Gamage and Mathew (2008) addressed the problem of testing the equal of sub-vectors of two multivariate normal mean vectors when the complementary subvectors are known to be equal. A test procedure is derived using the multivariate satterthwait approximation.

Imada (2011) obtained the test statistics and it's null distribution for a multivariate twosided test for testing wehther the noram mean vector is equal to zero or not. Bazyari and Chinipardaz (2013) considered the problem of testing the isotonic of several $p$-variate normal mean vectors against all alternatives. The test statistic proposed and a reformulation of the test statistic gave based on the orthogonal projections on the closed convex cones and then the upper bound for $p$-value of the test statistic computed. Bazyari and Pesarin (2013) considered testing the homogeneity of $k$ mean vectors against two-sided restricted alternatives separately in multivariate normal distributions. They examined the problem of testing under two separate cases. One case is that covariance matrices are known, the other one is that covariance matrices are unknown but common. When that covariance matrices are known the test statistic is obtained using the likelihood ratio method. The null distribution of test statistic is derived and its critical values are computed at different significance levels. A Monte Carlo study is also presented to estimate the power of the test. Bazyari (2015) extend Robertson and Wegman's (1978) problem to the multivariate normal distribution. They consider two different cases. One case is that covariance matrices are known, the other one is that they have an unknown scale factor. For both cases, they derive the test statistics and study their null distributions. The critical values and power of tests are estimated by Monte Carlo simulation study.

Bazyari (2017) considered testing homogeneity of multivariate normal mean vectors under an order restriction when the covariance matrices are unknown, arbitrary positive definite and unequal. When the covariance matrices are unknown and unequal, a bootstrap test statistic proposed and because of the main advantage of the bootstrap test is that it avoids the derivation of the complex null distribution analytically and is easy to implement, the bootstrap $p$-value defined and an algorithm presented to estimate it. Also, this test investigated more with respect to Type I error rates. Bazyari (2018) extended Bazyari and Chinipardaz's (2012) problem to multivariate case. He proposed an 
approximate test statistic based on orthogonal projections on the closed convex cones, studied its upper tail probability under the null hypothesis and estimate its critical values for different significance levels by using Monte Carlo simulation.

In the present paper, we consider the problem of testing given in (1) which for known covariance matrices is in fact, an extension of Kudo (1963) and Glimm et al. (2002) to several multivariate normal distributions. The extension to the multivariate normal distributions is important and of course interesting which to the authors' knowledge, the likelihood ratio test for this problem has not been obtained yet. The maximum likelihood estimate under the alternative hypothesis in our case also belongs to the parameters restricted category.

The rest of the paper is organized as follows. The test statistic based on the likelihood ratio criterion which has the general form under the restricted alternative hypothesis is obtained in Section 2. In Section 3, the main theorem concerning the maximum likelihood estimate under the one sided restricted alternative hypothesis with its proof is given. In Section 4, a geometric interpretation of the test statistic is discussed and also its null distribution obtained in the special case. In Section 5, we consider the case that covariance matrices are unknown and common. The critical values of the test statistic when the sample sizes are identical and also when they are different are estimated using Monte Carlo simulation in Section 6. Also, to evaluate the results, the effect of a drug is investigated on the body of one-day babies for suspected Sepsis disease.

\section{Computing the test statistic}

In this section, we prepare the likelihood ratio test for the problem of testing $\mathrm{H}_{0}$ versus $\mathrm{H}_{1}$. The likelihood function for $k \geq 2$ multivariate normal distributions is as follows

$$
\mathrm{L}\left(\boldsymbol{\mu}_{1}, \boldsymbol{\mu}_{2}, \ldots, \boldsymbol{\mu}_{\mathrm{k}}\right)=\prod_{\mathrm{i}=1}^{\mathrm{k}} \frac{1}{(2 \pi)^{\mathrm{p} / 2}}\left|\boldsymbol{\Sigma}_{\mathrm{i}}\right|^{-1 / 2} \cdot \exp \left\{-\frac{1}{2} \sum_{\mathrm{j}=1}^{\mathrm{n}_{\mathrm{i}}}\left(\mathbf{X}_{\mathrm{ij}}-\boldsymbol{\mu}_{\mathrm{i}}\right)^{\prime} \boldsymbol{\Sigma}_{\mathrm{i}}^{-1}\left(\mathbf{X}_{\mathrm{ij}}-\boldsymbol{\mu}_{\mathrm{i}}\right)\right\} \text {. }
$$

Then the likelihood ratio test statistic is

$$
\begin{aligned}
& \lambda= \frac{\sup _{\mathrm{H}_{0}} L\left(\boldsymbol{\mu}_{1}, \boldsymbol{\mu}_{2}, \ldots, \boldsymbol{\mu}_{\mathrm{k}}\right)}{\sup _{\mathrm{H}_{1}} L\left(\boldsymbol{\mu}_{1}, \boldsymbol{\mu}_{2}, \ldots, \boldsymbol{\mu}_{\mathrm{k}}\right)} \\
&= \sup _{\mathrm{H}_{0}} \prod_{\mathrm{i}=1}^{\mathrm{k}} \frac{1}{(2 \pi)^{\mathrm{p} / 2}}\left|\boldsymbol{\Sigma}_{\mathrm{i}}\right|^{-1 / 2} \cdot \exp \left\{-\frac{1}{2} \sum_{\mathrm{j}=1}^{\mathrm{n}_{\mathrm{i}}}\left(\mathbf{X}_{\mathrm{ij}}-\boldsymbol{\mu}_{\mathrm{i}}\right)^{\prime} \boldsymbol{\Sigma}_{\mathrm{i}}^{-1}\left(\mathbf{X}_{\mathrm{ij}}-\boldsymbol{\mu}_{\mathrm{i}}\right)\right\} \\
& \sup _{\mathrm{H}_{1}} \prod_{\mathrm{i}=1}^{\mathrm{k}} \frac{1}{(2 \pi)^{\mathrm{p} / 2}}\left|\boldsymbol{\Sigma}_{\mathrm{i}}\right|^{-1 / 2} \cdot \exp \left\{-\frac{1}{2} \sum_{\mathrm{j}=1}^{\mathrm{n}_{\mathrm{i}}}\left(\mathbf{X}_{\mathrm{ij}}-\boldsymbol{\mu}_{\mathrm{i}}\right)^{\prime} \boldsymbol{\Sigma}_{\mathrm{i}}^{-1}\left(\mathbf{X}_{\mathrm{ij}}-\boldsymbol{\mu}_{\mathrm{i}}\right)\right\}
\end{aligned}
$$

Now, since under the null hypothesis, the mean vectors are equal to zero, so we have that 
Then we get

$$
\begin{aligned}
& \sup _{\mathrm{H}_{0}} \prod_{\mathrm{i}=1}^{\mathrm{k}} \frac{1}{(2 \pi)^{\mathrm{p} / 2}}\left|\boldsymbol{\Sigma}_{\mathrm{i}}\right|^{-1 / 2} \cdot \exp \left\{-\frac{1}{2} \sum_{\mathrm{j}=1}^{\mathrm{n}_{\mathrm{i}}}\left(\mathbf{X}_{\mathrm{ij}}-\boldsymbol{\mu}_{\mathrm{i}}\right)^{\prime} \boldsymbol{\Sigma}_{\mathrm{i}}^{-1}\left(\mathbf{X}_{\mathrm{ij}}-\boldsymbol{\mu}_{\mathrm{i}}\right)\right\} \\
& =\prod_{\mathrm{i}=1}^{\mathrm{k}} \frac{1}{(2 \pi)^{\mathrm{p} / 2}}\left|\boldsymbol{\Sigma}_{\mathrm{i}}\right|^{-1 / 2} \cdot \exp \left\{-\frac{1}{2} \sum_{\mathrm{j}=1}^{\mathrm{n}_{\mathrm{i}}} \mathbf{X}_{\mathrm{ij}} \boldsymbol{\Sigma}_{\mathrm{i}}^{-1} \mathbf{X}_{\mathrm{ij}}\right\} .
\end{aligned}
$$

$$
\begin{aligned}
& \lambda=\frac{\prod_{\mathrm{i}=1}^{\mathrm{k}} \frac{1}{(2 \pi)^{\mathrm{p} / 2}}\left|\boldsymbol{\Sigma}_{\mathrm{i}}\right|^{-1 / 2} \cdot \exp \left\{-\frac{1}{2} \sum_{\mathrm{j}=1}^{\mathrm{n}_{\mathrm{i}}} \mathbf{X}_{\mathrm{ij}}^{\prime} \boldsymbol{\Sigma}_{\mathrm{i}}^{-1} \mathbf{X}_{\mathrm{ij}}\right\}}{\sup _{\mathrm{H}_{1}} \prod_{\mathrm{i}=1}^{\mathrm{k}} \frac{1}{(2 \pi)^{\mathrm{p} / 2}}\left|\boldsymbol{\Sigma}_{\mathrm{i}}\right|^{-1 / 2} \cdot \exp \left\{-\frac{1}{2} \sum_{\mathrm{j}=1}^{\mathrm{n}_{\mathrm{i}}}\left(\mathbf{X}_{\mathrm{ij}}-\boldsymbol{\mu}_{\mathrm{i}}\right)^{\prime} \boldsymbol{\Sigma}_{\mathrm{i}}^{-1}\left(\mathbf{X}_{\mathrm{ij}}-\boldsymbol{\mu}_{\mathrm{i}}\right)\right\}} \\
& =\frac{e^{-\frac{1}{2} \sum_{\mathrm{i}=1}^{\mathrm{k}} \sum_{\mathrm{j}=1}^{\mathrm{n}_{\mathrm{i}}} \mathbf{X}_{\mathrm{ij}}^{\prime} \boldsymbol{\Sigma}_{\mathrm{i}}^{-1} \mathbf{X}_{\mathrm{ij}}}}{\sup _{\mathrm{H}_{1}} \mathrm{e}^{-\frac{1}{2_{\mathrm{i}=1 \mathrm{j}=1}^{\mathrm{k}} \sum_{\mathrm{i}}^{\mathrm{n}}\left(\mathbf{X}_{\mathrm{ij}}-\boldsymbol{\mu}_{\mathrm{i}}\right)^{\prime} \boldsymbol{\Sigma}_{\mathrm{i}}^{-1}\left(\mathbf{X}_{\mathrm{ij}}-\boldsymbol{\mu}_{\mathrm{i}}\right)}},}
\end{aligned}
$$

The likelihood ratio test rejects the hypothesis $H_{0}$ for small values of $\lambda$. The large values of the statistic

$$
\mathrm{T}=-2 \ln \lambda=\sum_{\mathrm{i}=1}^{\mathrm{k}} \sum_{\mathrm{j}=1}^{\mathrm{n}_{\mathrm{i}}} \mathbf{X}_{\mathrm{ij}}^{\prime} \boldsymbol{\Sigma}_{\mathrm{i}}^{-1} \mathbf{X}_{\mathrm{ij}}-\min _{\mathrm{H}_{\mathrm{i}}} \sum_{\mathrm{i}=1}^{\mathrm{k}} \sum_{\mathrm{j}=1}^{\mathrm{n}_{\mathrm{i}}}\left(\mathbf{X}_{\mathrm{ij}}-\boldsymbol{\mu}_{\mathrm{i}}\right)^{\prime} \boldsymbol{\Sigma}_{\mathrm{i}}^{-1}\left(\mathbf{X}_{\mathrm{ij}}-\boldsymbol{\mu}_{\mathrm{i}}\right),
$$

reject $\mathrm{H}_{0}$. To compute the value of $\mathrm{T}$ statistic, we need merely find the minimum of term $\sum_{\mathrm{i}=1}^{\mathrm{k}} \sum_{\mathrm{j}=1}^{\mathrm{n}_{\mathrm{i}}}\left(\mathbf{X}_{\mathrm{ij}}-\boldsymbol{\mu}_{\mathrm{i}}\right)^{\prime} \boldsymbol{\Sigma}_{\mathrm{i}}^{-1}\left(\mathbf{X}_{\mathrm{ij}}-\boldsymbol{\mu}_{\mathrm{i}}\right)$ under the imposed condition on $\mathrm{H}_{1}$. In general, this can be done by quadratic programming (see Wolfe, 1959). They discussed about the minimization of a convex function of variables subject to linear inequalities. So, it is clear that there exists a vector $\boldsymbol{\mu}_{\mathrm{i}}^{*}$, such that

$$
\min _{\mathrm{H}_{1}} \sum_{\mathrm{i}=1}^{\mathrm{k}} \sum_{\mathrm{j}=1}^{\mathrm{n}_{\mathrm{i}}}\left(\mathbf{X}_{\mathrm{ij}}-\boldsymbol{\mu}_{\mathrm{i}}\right)^{\prime} \boldsymbol{\Sigma}_{\mathrm{i}}^{-1}\left(\mathbf{X}_{\mathrm{ij}}-\boldsymbol{\mu}_{\mathrm{i}}\right)=\sum_{\mathrm{i}=1}^{\mathrm{k}} \sum_{\mathrm{j}=1}^{\mathrm{n}_{\mathrm{i}}}\left(\mathbf{X}_{\mathrm{ij}}-\boldsymbol{\mu}_{\mathrm{i}}^{*}\right)^{\prime} \boldsymbol{\Sigma}_{\mathrm{i}}^{-1}\left(\mathbf{X}_{\mathrm{ij}}-\boldsymbol{\mu}_{\mathrm{i}}^{*}\right) \text {. }
$$

We note that, if there is not any restriction on the mean vector $\boldsymbol{\mu}_{\mathrm{i}}, i=1,2, \ldots, k$, then the estimator of $\boldsymbol{\mu}_{\mathrm{i}}$ is as follows. Suppose that for any $i, i=1,2, \ldots, k$, the vector $\hat{\boldsymbol{\mu}}_{\mathrm{i}}$ is an estimator for $\boldsymbol{\mu}_{\mathrm{i}}$. Then define the function $\mathrm{f}\left(\hat{\boldsymbol{\mu}}_{\mathrm{i}}\right)$ as

$$
\mathrm{f}\left(\hat{\boldsymbol{\mu}}_{\mathrm{i}}\right)=\sum_{\mathrm{i}=1}^{\mathrm{k}} \sum_{\mathrm{j}=1}^{\mathrm{n}_{\mathrm{i}}}\left(\mathbf{X}_{\mathrm{ij}}-\hat{\boldsymbol{\mu}}_{\mathrm{i}}\right)^{\prime} \boldsymbol{\Sigma}_{\mathrm{i}}^{-1}\left(\mathbf{X}_{\mathrm{ij}}-\hat{\boldsymbol{\mu}}_{\mathrm{i}}\right)=\sum_{\mathrm{i}=1}^{\mathrm{k}} \mathrm{g}_{\mathrm{i}},
$$

where $\mathrm{g}_{\mathrm{i}}=\sum_{\mathrm{j}=1}^{\mathrm{n}_{\mathrm{i}}}\left(\mathbf{X}_{\mathrm{ij}}-\hat{\boldsymbol{\mu}}_{\mathrm{i}}\right)^{\prime} \boldsymbol{\Sigma}_{\mathrm{i}}^{-1}\left(\mathbf{X}_{\mathrm{ij}}-\hat{\boldsymbol{\mu}}_{\mathrm{i}}\right)$. It is clear that

$$
\begin{aligned}
\mathrm{g}_{\mathrm{i}} & =\sum_{\mathrm{j}=1}^{\mathrm{n}_{\mathrm{i}}}\left(\mathbf{X}_{\mathrm{ij}}^{\prime} \boldsymbol{\Sigma}_{\mathrm{i}}^{-1} \mathbf{X}_{\mathrm{ij}}-\mathbf{X}_{\mathrm{ij}}^{\prime} \Sigma_{\mathrm{i}}^{-1} \hat{\boldsymbol{\mu}}_{\mathrm{i}}-\hat{\boldsymbol{\mu}}_{\mathrm{i}}^{\prime} \boldsymbol{\Sigma}_{\mathrm{i}}^{-1} \mathbf{X}_{\mathrm{ij}}+\hat{\boldsymbol{\mu}}_{\mathrm{i}}^{\prime} \boldsymbol{\Sigma}_{\mathrm{i}}^{-1} \hat{\boldsymbol{\mu}}_{\mathrm{i}}\right) \\
& =\sum_{\mathrm{j}=1}^{\mathrm{n}_{\mathrm{i}}}\left(\mathbf{X}_{\mathrm{ij}}^{\prime} \boldsymbol{\Sigma}_{\mathrm{i}}^{-1} \mathbf{X}_{\mathrm{ij}}-2 \hat{\boldsymbol{\mu}}_{\mathrm{i}}^{\prime} \boldsymbol{\Sigma}_{\mathrm{i}}^{-1} \mathbf{X}_{\mathrm{ij}}+\hat{\boldsymbol{\mu}}_{\mathrm{i}}^{\prime} \boldsymbol{\Sigma}_{\mathrm{i}}^{-1} \hat{\boldsymbol{\mu}}_{\mathrm{i}}\right)
\end{aligned}
$$

By differentiation of (3), with respect to the vector $\hat{\boldsymbol{\mu}}_{\mathrm{i}}$, we have 


$$
\mathrm{g}_{\mathrm{i}}^{\prime}=\sum_{j=1}^{n_{i}}\left(-2 \boldsymbol{\Sigma}_{\mathrm{i}}^{-1} \mathbf{X}_{\mathrm{ij}}+2 \boldsymbol{\Sigma}_{\mathrm{i}}^{-1} \hat{\boldsymbol{\mu}}_{\mathrm{i}}\right)
$$

Then

$$
\mathrm{f}\left(\hat{\boldsymbol{\mu}}_{\mathrm{i}}\right)=\sum_{\mathrm{j}=1}^{\mathrm{n}_{1}}\left(\mathbf{X}_{1 \mathrm{j}}-\hat{\boldsymbol{\mu}}_{\mathrm{i}}\right)^{\prime} \boldsymbol{\Sigma}_{1}^{-1}\left(\mathbf{X}_{1 \mathrm{j}}-\hat{\boldsymbol{\mu}}_{\mathrm{i}}\right)+\cdots+\sum_{\mathrm{j}=1}^{\mathrm{n}_{\mathrm{k}}}\left(\mathbf{X}_{\mathrm{kj}}-\hat{\boldsymbol{\mu}}_{\mathrm{i}}\right)^{\prime} \boldsymbol{\Sigma}_{\mathrm{k}}^{-1}\left(\mathbf{X}_{\mathrm{kj}}-\hat{\boldsymbol{\mu}}_{\mathrm{i}}\right) .
$$

Now, if $\left[\mathrm{f}\left(\hat{\boldsymbol{\mu}}_{\mathrm{i}}\right)\right]^{\prime}=0$, then it is clear that the maximum likelihood estimator of the parameter $\boldsymbol{\mu}_{\mathrm{i}}$ for any $i=1,2, \ldots, k$, when there is not any restriction on the mean vectors is $\overline{\mathbf{X}}_{\mathrm{i}}$.

\section{Main theorem concerning the maximum likelihood estimation}

In this section, our purpose is to find the conditions which under those the estimator $\boldsymbol{\mu}_{\mathrm{i}}^{*}$, be the maximum likelihood estimate under the alternative hypothesis. In the following theorem, we show that under the especial conditions, $\boldsymbol{\mu}_{\mathrm{i}}^{*}$ is the maximum likelihood estimate of $\boldsymbol{\mu}_{\mathrm{i}}$.

Theorem: A suitable condition for $\boldsymbol{\mu}_{\mathrm{i}}^{*}$ to be the maximum likelihood estimate is that for any $i=1,2, \ldots, k$, that one of the $i$ th components of the two vectors $\boldsymbol{\mu}_{\mathrm{i}}^{*}$ and $-\left(\boldsymbol{\Sigma}_{\mathrm{i}}^{-1}\left(\overline{\mathbf{X}}_{\mathrm{i}}-\boldsymbol{\mu}_{\mathrm{i}}^{*}\right)\right)$ is zero and the other is non-negative holds true.

Proof: If $\boldsymbol{\mu}_{\mathrm{i}}^{*}, i=1,2, \ldots, k$, be the maximum likelihood estimate, then it is necessary to have the following inequality for every mean vector $\boldsymbol{\mu}_{\mathrm{i}}$,

$$
\sum_{\mathrm{i}=1}^{\mathrm{k}} \sum_{\mathrm{j}=1}^{\mathrm{n}_{\mathrm{i}}}\left(\mathbf{X}_{\mathrm{ij}}-\boldsymbol{\mu}_{\mathrm{i}}\right)^{\prime} \boldsymbol{\Sigma}_{\mathrm{i}}^{-1}\left(\mathbf{X}_{\mathrm{ij}}-\boldsymbol{\mu}_{\mathrm{i}}\right)-\sum_{\mathrm{i}=1}^{\mathrm{k}} \sum_{\mathrm{j}=1}^{\mathrm{n}_{\mathrm{i}}}\left(\mathbf{X}_{\mathrm{ij}}-\boldsymbol{\mu}_{\mathrm{i}}^{*}\right)^{\prime} \boldsymbol{\Sigma}_{\mathrm{i}}^{-1}\left(\mathbf{X}_{\mathrm{ij}}-\boldsymbol{\mu}_{\mathrm{i}}^{*}\right) \geq 0 .
$$

First, we show that

$$
\begin{aligned}
& \sum_{\mathrm{i}=1}^{\mathrm{k}} \sum_{\mathrm{j}=1}^{\mathrm{n}_{\mathrm{i}}}\left(\mathbf{X}_{\mathrm{ij}}-\boldsymbol{\mu}_{\mathrm{i}}\right)^{\prime} \boldsymbol{\Sigma}_{\mathrm{i}}^{-1}\left(\mathbf{X}_{\mathrm{ij}}-\boldsymbol{\mu}_{\mathrm{i}}\right)-\sum_{\mathrm{i}=1}^{\mathrm{k}} \sum_{\mathrm{j}=1}^{\mathrm{n}_{\mathrm{i}}}\left(\mathbf{X}_{\mathrm{ij}}-\boldsymbol{\mu}_{\mathrm{i}}^{*}\right)^{\prime} \boldsymbol{\Sigma}_{\mathrm{i}}^{-1}\left(\mathbf{X}_{\mathrm{ij}}-\boldsymbol{\mu}_{\mathrm{i}}^{*}\right) \\
& =\sum_{\mathrm{i}=1}^{\mathrm{k}} \mathrm{n}_{\mathrm{i}}\left(\overline{\mathbf{X}}_{\mathrm{i}}-\boldsymbol{\mu}_{\mathrm{i}}\right)^{\prime} \boldsymbol{\Sigma}_{\mathrm{i}}^{-1}\left(\overline{\mathbf{X}}_{\mathrm{i}}-\boldsymbol{\mu}_{\mathrm{i}}\right)-\sum_{\mathrm{i}=1}^{\mathrm{k}} \mathrm{n}_{\mathrm{i}}\left(\overline{\mathbf{X}}_{\mathrm{i}}-\boldsymbol{\mu}_{\mathrm{i}}^{*}\right)^{\prime} \boldsymbol{\Sigma}_{\mathrm{i}}^{-1}\left(\overline{\mathbf{X}}_{\mathrm{i}}-\boldsymbol{\mu}_{\mathrm{i}}^{*}\right),
\end{aligned}
$$

So, we summarize each term

$$
\begin{aligned}
& \sum_{\mathrm{i}=1}^{\mathrm{k}} \sum_{\mathrm{j}=1}^{\mathrm{n}_{\mathrm{i}}}\left(\mathbf{X}_{\mathrm{ij}}-\boldsymbol{\mu}_{\mathrm{i}}\right)^{\prime} \boldsymbol{\Sigma}_{\mathrm{i}}^{-1}\left(\mathbf{X}_{\mathrm{ij}}-\boldsymbol{\mu}_{\mathrm{i}}\right) \\
& =\sum_{\mathrm{i}=1}^{\mathrm{k}} \sum_{\mathrm{j}=1}^{\mathrm{n}_{\mathrm{i}}}\left(\left(\mathbf{X}_{\mathrm{ij}}-\overline{\mathbf{X}}_{\mathrm{i}}\right)+\left(\overline{\mathbf{X}}_{\mathrm{i}}-\boldsymbol{\mu}_{\mathrm{i}}\right)\right) \boldsymbol{\Sigma}_{\mathrm{i}}^{-1}\left(\left(\mathbf{X}_{\mathrm{ij}}-\overline{\mathbf{X}}_{\mathrm{i}}\right)+\left(\overline{\mathbf{X}}_{\mathrm{i}}-\boldsymbol{\mu}_{\mathrm{i}}\right)\right) \\
& =\sum_{\mathrm{i}=1}^{\mathrm{k}} \sum_{\mathrm{j}=1}^{\mathrm{n}_{\mathrm{i}}}\left(\left(\mathbf{X}_{\mathrm{ij}}-\overline{\mathbf{X}}_{\mathrm{i}}\right)^{\prime} \boldsymbol{\Sigma}_{\mathrm{i}}^{-1}\left(\mathrm{X}_{\mathrm{ij}}-\overline{\mathbf{X}}_{\mathrm{i}}\right)+\left(\mathbf{X}_{\mathrm{ij}}-\overline{\mathbf{X}}_{\mathrm{i}}\right)^{\prime} \boldsymbol{\Sigma}_{\mathrm{i}}^{-1}\left(\overline{\mathbf{X}}_{\mathrm{i}}-\boldsymbol{\mu}_{\mathrm{i}}\right)\right. \\
& \left.\quad+\left(\overline{\mathbf{X}}_{\mathrm{i}}-\boldsymbol{\mu}_{\mathrm{i}}\right)^{\prime} \boldsymbol{\Sigma}_{\mathrm{i}}^{-1}\left(\mathbf{X}_{\mathrm{ij}}-\overline{\mathbf{X}}_{\mathrm{i}}\right)+\left(\overline{\mathbf{X}}_{\mathrm{i}}-\boldsymbol{\mu}_{\mathrm{i}}\right)^{\prime} \boldsymbol{\Sigma}_{\mathrm{i}}^{-1}\left(\overline{\mathbf{X}}_{\mathrm{i}}-\boldsymbol{\mu}_{\mathrm{i}}\right)\right) .
\end{aligned}
$$

But, since 


$$
\sum_{\mathrm{j}=1}^{\mathrm{n}_{\mathrm{i}}}\left(\mathbf{X}_{\mathrm{ij}}-\overline{\mathbf{X}}_{\mathrm{i}}\right)^{\prime}=0 \quad, \quad \sum_{\mathrm{j}=1}^{\mathrm{n}_{\mathrm{i}}}\left(\mathbf{X}_{\mathrm{ij}}-\overline{\mathbf{X}}_{\mathrm{i}}\right)=0 .
$$

Then we get

$$
\begin{aligned}
& \sum_{\mathrm{i}=1}^{\mathrm{k}} \sum_{\mathrm{j}=1}^{\mathrm{n}_{\mathrm{i}}}\left(\mathbf{X}_{\mathrm{ij}}-\boldsymbol{\mu}_{\mathrm{i}}\right)^{\prime} \boldsymbol{\Sigma}_{\mathrm{i}}^{-1}\left(\mathbf{X}_{\mathrm{ij}}-\boldsymbol{\mu}_{\mathrm{i}}\right) \\
& =\sum_{\mathrm{i}=1}^{\mathrm{k}} \sum_{\mathrm{j}=1}^{\mathrm{n}_{\mathrm{i}}}\left(\mathbf{X}_{\mathrm{ij}}-\overline{\mathbf{X}}_{\mathrm{i}}\right)^{\prime} \boldsymbol{\Sigma}_{\mathrm{i}}^{-1}\left(\mathbf{X}_{\mathrm{ij}}-\overline{\mathbf{X}}_{\mathrm{i}}\right)+\sum_{\mathrm{i}=1}^{\mathrm{k}} \sum_{\mathrm{j}=1}^{\mathrm{n}_{\mathrm{i}}}\left(\overline{\mathbf{X}}_{\mathrm{i}}-\boldsymbol{\mu}_{\mathrm{i}}\right)^{\prime} \boldsymbol{\Sigma}_{\mathrm{i}}^{-1}\left(\overline{\mathbf{X}}_{\mathrm{i}}-\boldsymbol{\mu}_{\mathrm{i}}\right) \\
& =\sum_{\mathrm{i}=1}^{\mathrm{k}} \sum_{\mathrm{j}=1}^{\mathrm{n}_{\mathrm{i}}}\left(\mathbf{X}_{\mathrm{ij}}-\overline{\mathbf{X}}_{\mathrm{i}}\right)^{\prime} \boldsymbol{\Sigma}_{\mathrm{i}}^{-1}\left(\mathbf{X}_{\mathrm{ij}}-\overline{\mathbf{X}}_{\mathrm{i}}\right)+\sum_{\mathrm{i}=1}^{\mathrm{k}} \mathrm{n}_{\mathrm{i}}\left(\overline{\mathbf{X}}_{\mathrm{i}}-\boldsymbol{\mu}_{\mathrm{i}}\right)^{\prime} \boldsymbol{\Sigma}_{\mathrm{i}}^{-1}\left(\overline{\mathbf{X}}_{\mathrm{i}}-\boldsymbol{\mu}_{\mathrm{i}}\right) .
\end{aligned}
$$

Also we summarize the second term of formula (4) as follows, which the process is similar to the first term

$$
\begin{aligned}
& \sum_{\mathrm{i}=1}^{\mathrm{k}} \sum_{\mathrm{j}=1}^{\mathrm{n}_{\mathrm{i}}}\left(\mathbf{X}_{\mathrm{ij}}-\boldsymbol{\mu}_{\mathrm{i}}^{*}\right)^{\prime} \boldsymbol{\Sigma}_{\mathrm{i}}^{-1}\left(\mathbf{X}_{\mathrm{ij}}-\boldsymbol{\mu}_{\mathrm{i}}^{*}\right) \\
& =\sum_{\mathrm{i}=1}^{\mathrm{k}} \sum_{\mathrm{j}=1}^{\mathrm{n}_{\mathrm{i}}}\left(\mathbf{X}_{\mathrm{ij}}-\overline{\mathbf{X}}_{\mathrm{i}}\right)^{\prime} \boldsymbol{\Sigma}_{\mathrm{i}}^{-1}\left(\mathrm{X}_{\mathrm{ij}}-\overline{\mathbf{X}}_{\mathrm{i}}\right)+\sum_{\mathrm{i}=1}^{\mathrm{k}} \mathrm{n}_{\mathrm{i}}\left(\overline{\mathbf{X}}_{\mathrm{i}}-\boldsymbol{\mu}_{\mathrm{i}}^{*}\right)^{\prime} \boldsymbol{\Sigma}_{\mathrm{i}}^{-1}\left(\overline{\mathbf{X}}_{\mathrm{i}}-\boldsymbol{\mu}_{\mathrm{i}}^{*}\right) .
\end{aligned}
$$

Therefore

$$
\begin{gathered}
\sum_{\mathrm{i}=1}^{\mathrm{k}} \sum_{\mathrm{j}=1}^{\mathrm{n}_{\mathrm{i}}}\left(\mathbf{X}_{\mathrm{ij}}-\boldsymbol{\mu}_{\mathrm{i}}\right)^{\prime} \boldsymbol{\Sigma}_{\mathrm{i}}^{-1}\left(\mathbf{X}_{\mathrm{ij}}-\boldsymbol{\mu}_{\mathrm{i}}\right)-\sum_{\mathrm{i}=1}^{\mathrm{k}} \sum_{\mathrm{j}=1}^{\mathrm{n}_{\mathrm{i}}}\left(\mathbf{X}_{\mathrm{ij}}-\boldsymbol{\mu}_{\mathrm{i}}^{*}\right)^{\prime} \boldsymbol{\Sigma}_{\mathrm{i}}^{-1}\left(\mathbf{X}_{\mathrm{ij}}-\boldsymbol{\mu}_{\mathrm{i}}^{*}\right) \\
=\sum_{\mathrm{i}=1}^{\mathrm{k}} \mathrm{n}_{\mathrm{i}}\left(\overline{\mathbf{X}}_{\mathrm{i}}-\boldsymbol{\mu}_{\mathrm{i}}\right)^{\prime} \boldsymbol{\Sigma}_{\mathrm{i}}^{-1}\left(\overline{\mathbf{X}}_{\mathrm{i}}-\boldsymbol{\mu}_{\mathrm{i}}\right)-\sum_{\mathrm{i}=1}^{\mathrm{k}} \mathrm{n}_{\mathrm{i}}\left(\overline{\mathbf{X}}_{\mathrm{i}}-\boldsymbol{\mu}_{\mathrm{i}}^{*}\right)^{\prime} \boldsymbol{\Sigma}_{\mathrm{i}}^{-1}\left(\overline{\mathbf{X}}_{\mathrm{i}}-\boldsymbol{\mu}_{\mathrm{i}}^{*}\right) \\
=\sum_{\mathrm{i}=1}^{\mathrm{k}} \mathrm{n}_{\mathrm{i}}\left(\overline{\mathbf{X}}_{\mathrm{i}}^{\prime} \boldsymbol{\Sigma}_{\mathrm{i}}^{-1} \overline{\mathbf{X}}_{\mathrm{i}}-\overline{\mathbf{X}}_{\mathrm{i}}^{\prime} \boldsymbol{\Sigma}_{\mathrm{i}}^{-1} \boldsymbol{\mu}_{\mathrm{i}}-\boldsymbol{\mu}_{\mathrm{i}}^{\prime} \boldsymbol{\Sigma}_{\mathrm{i}}^{-1} \overline{\mathbf{X}}_{\mathrm{i}}+\boldsymbol{\mu}_{\mathrm{i}}^{\prime} \boldsymbol{\Sigma}_{\mathrm{i}}^{-1} \boldsymbol{\mu}_{\mathrm{i}}\right. \\
\left.\quad-\overline{\mathbf{X}}_{\mathrm{i}}^{\prime} \boldsymbol{\Sigma}_{\mathrm{i}}^{-1} \overline{\mathbf{X}}_{\mathrm{i}}+\overline{\mathbf{X}}_{\mathrm{i}}^{\prime} \boldsymbol{\Sigma}_{\mathrm{i}}^{-1} \boldsymbol{\mu}_{\mathrm{i}}^{*}+\boldsymbol{\mu}_{\mathrm{i}}^{*} \boldsymbol{\Sigma}_{\mathrm{i}}^{-1} \overline{\mathbf{X}}_{\mathrm{i}}-\boldsymbol{\mu}_{\mathrm{i}}^{*} \boldsymbol{\Sigma}_{\mathrm{i}}^{-1} \boldsymbol{\mu}_{\mathrm{i}}^{*}\right) \\
=\sum_{\mathrm{i}=1}^{\mathrm{k}} \mathrm{n}_{\mathrm{i}}\left(\boldsymbol{\mu}_{\mathrm{i}}^{\prime} \boldsymbol{\Sigma}_{\mathrm{i}}^{-1} \boldsymbol{\mu}_{\mathrm{i}}-\boldsymbol{\mu}_{\mathrm{i}}^{*} \boldsymbol{\Sigma}_{\mathrm{i}}^{-1} \boldsymbol{\mu}_{\mathrm{i}}^{*}-2 \boldsymbol{\mu}_{\mathrm{i}}^{\prime} \boldsymbol{\Sigma}_{\mathrm{i}}^{-1} \overline{\mathbf{X}}_{\mathrm{i}}+2 \boldsymbol{\mu}_{\mathrm{i}}^{\prime} \boldsymbol{\Sigma}_{\mathrm{i}}^{-1} \overline{\mathbf{X}}_{\mathrm{i}}\right),
\end{gathered}
$$

Now, with paying attention to

$$
\begin{aligned}
& \sum_{\mathrm{i}=1}^{\mathrm{k}} \mathrm{n}_{\mathrm{i}}\left(\boldsymbol{\mu}_{\mathrm{i}}^{\prime} \boldsymbol{\Sigma}_{\mathrm{i}}^{-1} \boldsymbol{\mu}_{\mathrm{i}}-\boldsymbol{\mu}_{\mathrm{i}}^{*} \boldsymbol{\Sigma}_{\mathrm{i}}^{-1} \boldsymbol{\mu}_{\mathrm{i}}^{*}-2 \boldsymbol{\mu}_{\mathrm{i}}^{\prime} \boldsymbol{\Sigma}_{\mathrm{i}}^{-1} \overline{\mathbf{X}}_{\mathrm{i}}+2 \boldsymbol{\mu}_{\mathrm{i}}^{\prime} \boldsymbol{\Sigma}_{\mathrm{i}}^{-1} \overline{\mathbf{X}}_{\mathrm{i}}\right) \\
& =\sum_{\mathrm{i}=1}^{\mathrm{k}} \mathrm{n}_{\mathrm{i}}\left(\boldsymbol{\mu}_{\mathrm{i}}^{\prime} \boldsymbol{\Sigma}_{\mathrm{i}}^{-1} \boldsymbol{\mu}_{\mathrm{i}}+\boldsymbol{\mu}_{\mathrm{i}}^{\prime} \boldsymbol{\Sigma}_{\mathrm{i}}^{-1} \boldsymbol{\mu}_{\mathrm{i}}^{*}-\boldsymbol{\mu}_{\mathrm{i}}^{*} \boldsymbol{\Sigma}_{\mathrm{i}}^{-1} \boldsymbol{\mu}_{\mathrm{i}}-\boldsymbol{\mu}_{\mathrm{i}}^{*} \boldsymbol{\Sigma}_{\mathrm{i}}^{-1} \boldsymbol{\mu}_{\mathrm{i}}^{*}-2 \boldsymbol{\mu}_{\mathrm{i}}^{\prime} \boldsymbol{\Sigma}_{\mathrm{i}}^{-1} \overline{\mathbf{X}}_{\mathrm{i}}+2 \boldsymbol{\mu}_{\mathrm{i}}^{*} \boldsymbol{\Sigma}_{\mathrm{i}}^{-1} \overline{\mathbf{X}}_{\mathrm{i}}\right) \\
& =\sum_{\mathrm{i}=1}^{\mathrm{k}} \mathrm{n}_{\mathrm{i}}\left(\boldsymbol{\mu}_{\mathrm{i}}^{\prime} \boldsymbol{\Sigma}_{\mathrm{i}}^{-1} \boldsymbol{\mu}_{\mathrm{i}}-\boldsymbol{\mu}_{\mathrm{i}}^{\prime} \boldsymbol{\Sigma}_{\mathrm{i}}^{-1} \boldsymbol{\mu}_{\mathrm{i}}^{*}-\boldsymbol{\mu}_{\mathrm{i}}^{*} \boldsymbol{\Sigma}_{\mathrm{i}}^{-1} \boldsymbol{\mu}_{\mathrm{i}}+\boldsymbol{\mu}_{\mathrm{i}}^{*} \boldsymbol{\Sigma}_{\mathrm{i}}^{-1} \boldsymbol{\mu}_{\mathrm{i}}^{*}\right. \\
& \left.\quad-2 \boldsymbol{\mu}_{\mathrm{i}}^{\prime} \boldsymbol{\Sigma}_{\mathrm{i}}^{-1} \overline{\mathbf{X}}_{\mathrm{i}}+2 \boldsymbol{\mu}_{\mathrm{i}}^{\prime} \boldsymbol{\Sigma}_{\mathrm{i}}^{-1} \boldsymbol{\mu}_{\mathrm{i}}^{*}+2 \boldsymbol{\mu}_{\mathrm{i}}^{*^{\prime}} \boldsymbol{\Sigma}_{\mathrm{i}}^{-1} \overline{\mathbf{X}}_{\mathrm{i}}-2 \boldsymbol{\mu}_{\mathrm{i}}^{*} \boldsymbol{\Sigma}_{\mathrm{i}}^{-1} \boldsymbol{\mu}_{\mathrm{i}}^{*}\right) \\
& =\sum_{\mathrm{i}=1}^{\mathrm{k}} \mathrm{n}_{\mathrm{i}}\left(\boldsymbol{\mu}_{\mathrm{i}}-\boldsymbol{\mu}_{\mathrm{i}}^{*}\right)^{\prime} \boldsymbol{\Sigma}_{\mathrm{i}}^{-1}\left(\boldsymbol{\mu}_{\mathrm{i}}-\boldsymbol{\mu}_{\mathrm{i}}^{*}\right)-2 \sum_{\mathrm{i}=1}^{\mathrm{k}} \mathrm{n}_{\mathrm{i}}\left(\boldsymbol{\mu}_{\mathrm{i}}-\boldsymbol{\mu}_{\mathrm{i}}^{*}\right)^{\prime} \boldsymbol{\Sigma}_{\mathrm{i}}^{-1}\left(\overline{\mathbf{X}}_{\mathrm{i}}-\boldsymbol{\mu}_{\mathrm{i}}^{*}\right) .
\end{aligned}
$$

So, we should show that 


$$
\sum_{\mathrm{i}=1}^{\mathrm{k}} \mathrm{n}_{\mathrm{i}}\left(\boldsymbol{\mu}_{\mathrm{i}}-\boldsymbol{\mu}_{\mathrm{i}}^{*}\right)^{\prime} \boldsymbol{\Sigma}_{\mathrm{i}}^{-1}\left(\boldsymbol{\mu}_{\mathrm{i}}-\boldsymbol{\mu}_{\mathrm{i}}^{*}\right)-2 \sum_{\mathrm{i}=1}^{\mathrm{k}} \mathrm{n}_{\mathrm{i}}\left(\boldsymbol{\mu}_{\mathrm{i}}-\boldsymbol{\mu}_{\mathrm{i}}^{*}\right)^{\prime} \boldsymbol{\Sigma}_{\mathrm{i}}^{-1}\left(\overline{\mathbf{X}}_{\mathrm{i}}-\boldsymbol{\mu}_{\mathrm{i}}^{*}\right) \geq 0 .
$$

From the condition it follows that for any $i=1,2, \ldots, k$, one of the $i$ th components of the two vectors $\boldsymbol{\mu}_{\mathrm{i}}^{*}$ and $-\left\{\boldsymbol{\Sigma}_{\mathrm{i}}^{-1}\left(\overline{\mathbf{X}}_{\mathrm{i}}-\boldsymbol{\mu}_{\mathrm{i}}^{*}\right)\right\}$ is zero and the other is non-negative. Since, first suppose that for any $i=1,2, \ldots, k, i$ th component of vector $-\left\{\Sigma_{\mathrm{i}}^{-1}\left(\overline{\mathbf{X}}_{\mathrm{i}}-\boldsymbol{\mu}_{\mathrm{i}}^{*}\right)\right\}$ is zero, then it is clear that

$$
-2 \sum_{\mathrm{i}=1}^{\mathrm{k}} \mathrm{n}_{\mathrm{i}}\left(\boldsymbol{\mu}_{\mathrm{i}}-\boldsymbol{\mu}_{\mathrm{i}}^{*}\right)^{\prime} \boldsymbol{\Sigma}_{\mathrm{i}}^{-1}\left(\overline{\mathbf{X}}_{\mathrm{i}}-\boldsymbol{\mu}_{\mathrm{i}}^{*}\right)=0
$$

On the other hand, since for any $i=1,2, \ldots, k, \Sigma_{\mathrm{i}}$ is a positive definite, therefore

$$
\left(\boldsymbol{\mu}_{\mathrm{i}}-\boldsymbol{\mu}_{\mathrm{i}}^{*}\right)^{\prime} \boldsymbol{\Sigma}_{\mathrm{i}}^{-1}\left(\boldsymbol{\mu}_{\mathrm{i}}-\boldsymbol{\mu}_{\mathrm{i}}^{*}\right) \geq 0
$$

and the result is clear.

But if we suppose that for any $i=1,2, \ldots, k$, i th component of vector $-\left\{\boldsymbol{\Sigma}_{\mathrm{i}}^{-1}\left(\overline{\mathbf{X}}_{\mathrm{i}}-\boldsymbol{\mu}_{\mathrm{i}}^{*}\right)\right\}$ is non-negative, since $\boldsymbol{\mu}_{\mathrm{i}}^{*}=\mathbf{0}$, so that $\boldsymbol{\mu}_{\mathrm{i}}-\boldsymbol{\mu}_{\mathrm{i}}^{*} \geq \mathbf{0}$ and with paying attention to the inequality $\left(\boldsymbol{\mu}_{\mathrm{i}}-\boldsymbol{\mu}_{\mathrm{i}}^{*}\right)^{\prime} \boldsymbol{\Sigma}_{\mathrm{i}}^{-1}\left(\boldsymbol{\mu}_{\mathrm{i}}-\boldsymbol{\mu}_{\mathrm{i}}^{*}\right) \geq 0$, the result is clear.

\section{Geometric interpretation of the test statistic}

In this section a geometric interpretation of the test statistic is given. As for any $i=1,2, \ldots, k, \boldsymbol{\Sigma}_{\mathrm{i}}$ is a non-singular symmetric positive definite matrix, there exists a nonsingular matrix $\mathbf{A}_{\mathrm{i}}=\left(\mathrm{a}_{\mathrm{ij}}\right)$, such that

$$
\mathbf{A}_{\mathrm{i}} \boldsymbol{\Sigma}_{\mathrm{i}} \mathbf{A}_{i}^{\prime}=\mathbf{I}
$$

where $\mathbf{I}$ is the unit matrix. Now, define the variable $\overline{\mathbf{Y}}_{\mathrm{i}}=\mathbf{A}_{\mathrm{i}} \overline{\mathbf{X}}_{\mathrm{i}}$, then we have that,

$$
\begin{aligned}
\overline{\mathbf{X}}_{\mathrm{i}}=\mathbf{A}_{\mathrm{i}}^{-1} \overline{\mathbf{Y}}_{\mathrm{i}} \text { and } \overline{\mathbf{X}}_{\mathrm{i}}^{\prime}=\overline{\mathbf{Y}}_{\mathrm{i}}^{\prime} \mathbf{A}_{\mathrm{i}}^{-1} \text {. So } \\
\qquad \mathrm{T}^{*}=\sum_{\mathrm{i}=1}^{\mathrm{k}} \mathrm{n}_{\mathrm{i}} \overline{\mathbf{Y}}_{\mathrm{i}} \overline{\mathbf{Y}}_{\mathrm{i}}-\sum_{\mathrm{i}=1}^{\mathrm{k}} \mathrm{n}_{\mathrm{i}}\left(\overline{\mathbf{Y}}_{\mathrm{i}}-\mathbf{m}_{\mathrm{i}}\right)^{\prime} \boldsymbol{\Sigma}_{\mathrm{i}}^{-1}\left(\overline{\mathbf{Y}}_{\mathrm{i}}-\mathbf{m}_{\mathrm{i}}\right),
\end{aligned}
$$

where $\mathbf{m}_{\mathrm{i}}=\mathbf{A}_{\mathrm{i}} \boldsymbol{\mu}_{\mathrm{i}}$. Therefore $\mathrm{T}^{*}$ is the difference between the sum of the square of length of the vector $\overline{\mathbf{Y}}_{\mathrm{i}}$ in the space of $\mathbf{m}_{1}, \mathbf{m}_{2}, \ldots, \mathbf{m}_{\mathrm{k}}$ and sum of the square of distance between a point $\overline{\mathbf{Y}}_{\mathrm{i}}$ and a closed convex polyhedral cone $c$, defined by the inequalities

$$
\mu_{\mathrm{ij}}=\sum_{\mathrm{j}=1}^{\mathrm{p}} \mathrm{a}^{\mathrm{ij}} \mathrm{m}_{\mathrm{ij}} \geq 0
$$

where $\mu_{\mathrm{ij}}$ and $\mathrm{m}_{\mathrm{ij}}, j=1,2, \ldots, p$, are the $j$ th components of the vectors $\boldsymbol{\mu}_{\mathrm{i}}$ and $\mathbf{m}_{\mathrm{i}}$ respectively and we define the inverse of $\mathbf{A}_{\mathrm{i}}$ as $\mathbf{A}_{\mathrm{i}}^{-1}=\left(\mathrm{a}^{\mathrm{ij}}\right)$.

\subsection{The null distribution of the test statistic}

In this subsection, we derive the null distribution of the test statistic in the special case. If for any $i=1,2, \ldots, k, \overline{\mathbf{Y}}_{\mathrm{i}}$ is in the convex polyhedral convex $\mathrm{C}$, the second term of formula (6) vanishes and we have 


$$
\mathrm{T}^{*}=\sum_{\mathrm{i}=1}^{\mathrm{k}} \mathrm{n}_{\mathrm{i}} \overline{\mathbf{Y}}_{\mathrm{i}}^{\prime} \overline{\mathbf{Y}}_{\mathrm{i}}=\sum_{\mathrm{i}=1}^{\mathrm{k}} \overline{\mathbf{X}}_{\mathrm{i}}^{\prime}\left(\frac{\boldsymbol{\Sigma}_{\mathrm{i}}}{\mathrm{n}_{\mathrm{i}}}\right)^{-1} \overline{\mathbf{X}}_{\mathrm{i}}
$$

and it is easy to compute the value of the test statistic. To compute the null distribution of the statistic, since for any $i=1,2, \ldots, k$, the matrix $\left(\frac{\boldsymbol{\Sigma}_{\mathrm{i}}}{\mathrm{n}_{\mathrm{i}}}\right)^{-1}$ is a symmetric matrix and under the null hypothesis, $\overline{\mathbf{X}}_{\mathrm{i}}$ is distributed as $\mathrm{N}_{\mathrm{p}}\left(\mathbf{0}, \frac{\boldsymbol{\Sigma}_{\mathrm{i}}}{\mathrm{n}_{\mathrm{i}}}\right)$, then the distribution of $\overline{\mathbf{X}}_{\mathrm{i}}^{\prime}\left(\frac{\boldsymbol{\Sigma}_{\mathrm{i}}}{\mathrm{n}_{\mathrm{i}}}\right)^{-1} \overline{\mathbf{X}}_{\mathrm{i}}$ is $\chi_{\mathrm{p}}^{2}$ with $p$ degrees of freedom. Also since for any $i, i=1,2, \ldots, k$, $\overline{\mathbf{X}}_{\mathrm{i}}^{\prime}\left(\frac{\boldsymbol{\Sigma}_{\mathrm{i}}}{\mathrm{n}_{\mathrm{i}}}\right)^{-1} \overline{\mathbf{X}}_{\mathrm{i}}{ }^{\prime}$ s are independent variables, so the test statistic given in (7) is distributed as $\chi_{k p}^{2}$ with $k p$ degrees of freedom.

\section{Test statistic with unknown and common covariance matrices}

In this section, we suppose that the covariance matrices are completely unknown but common. We consider the problem of testing $\mathrm{H}_{0}$ against $\mathrm{H}_{1}$, where $\mathrm{H}_{1}$ is defined as before. Then the likelihood function is

$$
\begin{aligned}
\mathrm{L}(\boldsymbol{\mu}) & =\prod_{\mathrm{i}=1}^{\mathrm{k}} \frac{1}{(2 \pi)^{\mathrm{p} / 2}}|\boldsymbol{\Sigma}|^{-1 / 2} \cdot \exp \left\{-\frac{1}{2} \sum_{\mathrm{j}=1}^{\mathrm{n}_{\mathrm{i}}}\left(\mathbf{X}_{\mathrm{ij}}-\boldsymbol{\mu}_{\mathrm{i}}\right)^{\prime} \boldsymbol{\Sigma}^{-1}\left(\mathbf{X}_{\mathrm{ij}}-\boldsymbol{\mu}_{\mathrm{i}}\right)\right\} \\
& =\mathrm{c}|\boldsymbol{\Sigma}|^{-\mathrm{k} / 2} \exp \left\{-\frac{1}{2}\left(\sum_{\mathrm{i}=1}^{\mathrm{k}} \sum_{\mathrm{j}=1}^{\mathrm{n}_{\mathrm{i}}}\left(\mathbf{X}_{\mathrm{ij}}-\boldsymbol{\mu}_{\mathrm{i}}\right)^{\prime} \boldsymbol{\Sigma}^{-1}\left(\mathbf{X}_{\mathrm{ij}}-\boldsymbol{\mu}_{\mathrm{i}}\right)\right)\right\},
\end{aligned}
$$

where $\mathrm{c}$ is a positive constant which is independent of parameters $\boldsymbol{\mu}_{\mathrm{i}}$ and $\boldsymbol{\Sigma}$. The likelihood ratio test statistic is

$$
\lambda^{\prime}=\frac{\sup _{\boldsymbol{\mu} \in \mathrm{H}_{0}, \boldsymbol{\Sigma}} \mathrm{L}(\boldsymbol{\mu})}{\sup _{\boldsymbol{\mu} \in \mathrm{H}_{1}, \boldsymbol{\Sigma}} \mathrm{L}(\boldsymbol{\mu})},
$$

and since $\sup _{\Sigma}$ is not dependent on $\sup _{\boldsymbol{\mu} \in \mathrm{H}_{0}}$ and $\sup _{\boldsymbol{\mu} \in \mathrm{H}_{1}}$, so we have

$$
\lambda^{\prime}=\frac{\sup _{\boldsymbol{\Sigma}} \sup _{\boldsymbol{\mu} \in \mathrm{H}_{0}} \mathrm{~L}(\boldsymbol{\mu})}{\sup \sup _{\boldsymbol{\mu} \in \mathrm{H}_{1}} \mathrm{~L}(\boldsymbol{\mu})},
$$

where $\sup _{\boldsymbol{\mu} \in \mathrm{H}_{0}}$ denotes the supremum for the parameters $\boldsymbol{\mu}_{1}, \ldots, \boldsymbol{\mu}_{\mathrm{k}}$ under $\mathrm{H}_{0}$ and $\sup _{\boldsymbol{\Sigma}}$ denotes the supremum for all the $p \times p$ positive definite matrices. Therefore, we have 


$$
\begin{aligned}
& \lambda^{\prime}= \sup _{\Sigma} \sup _{\boldsymbol{\mu} \in \mathrm{H}_{0}}|\boldsymbol{\Sigma}|^{-\mathrm{k} / 2} \exp \left\{-\frac{1}{2} \sum_{\mathrm{i}=1}^{\mathrm{k}} \sum_{\mathrm{j}=1}^{\mathrm{n}_{\mathrm{i}}}\left(\mathbf{X}_{\mathrm{ij}}-\boldsymbol{\mu}_{\mathrm{i}}\right)^{\prime} \boldsymbol{\Sigma}^{-1}\left(\mathbf{X}_{\mathrm{ij}}-\boldsymbol{\mu}_{\mathrm{i}}\right)\right\} \\
& \sup _{\Sigma} \sup _{\boldsymbol{\mu} \in \mathrm{H}_{1}}|\boldsymbol{\Sigma}|^{-\mathrm{k} / 2} \exp \left\{-\frac{1}{2} \sum_{\mathrm{i}=1}^{\mathrm{k}} \sum_{\mathrm{j}=1}^{\mathrm{n}_{\mathrm{i}}}\left(\mathbf{X}_{\mathrm{ij}}-\boldsymbol{\mu}_{\mathrm{i}}\right)^{\prime} \boldsymbol{\Sigma}^{-1}\left(\mathbf{X}_{\mathrm{ij}}-\boldsymbol{\mu}_{\mathrm{i}}\right)\right\} \\
&=\frac{\left|\hat{\boldsymbol{\Sigma}}_{0}\right|^{-\mathrm{k} / 2} \mathrm{e}^{-\frac{1}{2} \sum_{\mathrm{i}=1}^{\mathrm{k}} \sum_{\mathrm{j}=1}^{\mathrm{n}_{\mathrm{i}}} \mathbf{X}_{\mathrm{ij}}^{\prime} \boldsymbol{j}_{0}^{-1} \mathbf{X}_{\mathrm{ij}}}}{\left|\hat{\boldsymbol{\Sigma}}_{1}\right|^{-\mathrm{k} / 2} \sup _{\mathrm{H}_{1}} \mathrm{e}^{-\frac{1}{2_{\mathrm{i}=1 \mathrm{j}=1}^{k} \sum_{\mathrm{i}}^{\mathrm{n}}\left(\mathbf{X}_{\mathrm{ij}}-\boldsymbol{\mu}_{\mathrm{i}}\right)^{\prime} \mathbf{\Sigma}_{1}^{-1}\left(\mathbf{X}_{\mathrm{ij}}-\boldsymbol{\mu}_{\mathrm{i}}\right)}}}
\end{aligned}
$$

where $\hat{\boldsymbol{\Sigma}}_{0}$ and $\hat{\boldsymbol{\Sigma}}_{1}$ are the estimators of the unknown matrix $\boldsymbol{\Sigma}$ under the hypotheses $\mathrm{H}_{0}$ and $\mathrm{H}_{1}$ respectively. So, we can write

$$
\begin{aligned}
-2 \ln \lambda^{\prime}= & \left(\operatorname{kln}\left|\hat{\boldsymbol{\Sigma}}_{0}\right|+\sum_{\mathrm{i}=1}^{\mathrm{k}} \sum_{\mathrm{j}=1}^{\mathrm{n}_{\mathrm{i}}} \mathbf{X}_{\mathrm{ij}}^{\prime} \boldsymbol{\Sigma}_{0}^{-1} \mathbf{X}_{\mathrm{ij}}\right) \\
& -\left(\operatorname{kln}\left|\hat{\boldsymbol{\Sigma}}_{1}\right|+\sum_{\mathrm{i}=1}^{\mathrm{k}} \sum_{\mathrm{j}=1}^{\mathrm{n}_{\mathrm{i}}}\left(\mathbf{X}_{\mathrm{ij}}-\boldsymbol{\mu}_{\mathrm{i}}^{*}\right)^{\prime} \boldsymbol{\Sigma}_{1}^{-1}\left(\mathbf{X}_{\mathrm{ij}}-\boldsymbol{\mu}_{\mathrm{i}}^{*}\right)\right) \\
= & \left(\operatorname{kln}\left|\hat{\boldsymbol{\Sigma}}_{0}\right|+\operatorname{tr} \sum_{\mathrm{i}=1}^{\mathrm{k}} \sum_{\mathrm{j}=1}^{\mathrm{n}_{\mathrm{i}}} \hat{\Sigma}_{0}^{-1} \mathbf{X}_{\mathrm{ij}}^{\prime} \mathbf{X}_{\mathrm{ij}}\right) \\
& -\left(\operatorname{kln}\left|\hat{\boldsymbol{\Sigma}}_{1}\right|+\operatorname{tr} \sum_{\mathrm{i}=1}^{\mathrm{k}} \hat{\boldsymbol{\Sigma}}_{1}^{-1} \sum_{\mathrm{j}=1}^{\mathrm{n}_{\mathrm{i}}}\left(\mathbf{X}_{\mathrm{ij}}-\boldsymbol{\mu}_{\mathrm{i}}^{*}\right)^{\prime}\left(\mathbf{X}_{\mathrm{ij}}-\boldsymbol{\mu}_{\mathrm{i}}^{*}\right)\right)
\end{aligned}
$$

where the symbol $t r$ denotes the trace of matrix and $\boldsymbol{\mu}_{\mathrm{i}}^{*}, i=1,2, \ldots, k$, is defined as before. Now, using lemma 3.2.2 of Anderson (1984) in the way similar to that of Anderson [(1984), Section 8.8], in order to get the likelihood ratio test for our problem we need to minimize the determinant

$$
\left|\mathbf{S}+\sum_{\mathrm{i}=1}^{\mathrm{k}} \mathrm{n}_{\mathrm{i}} \boldsymbol{\mu}_{\mathrm{i}} \boldsymbol{\mu}_{\mathrm{i}}^{\prime}\right|
$$

under the order hypothesis $\boldsymbol{\mu}_{1} \geq 0, \boldsymbol{\mu}_{2} \geq 0, \ldots, \boldsymbol{\mu}_{\mathrm{k}} \geq 0$, where we define $\mathbf{S}=\sum_{\mathrm{i}=1}^{\mathrm{k}} \sum_{\mathrm{j}=1}^{\mathrm{n}_{\mathrm{i}}} \mathbf{X}_{\mathrm{ij}} \mathbf{X}_{\mathrm{ij}}^{\prime}$, which under the null hypothesis is distributed with the Wishart distribution $\mathrm{W}_{\mathrm{p}}(\mathrm{N}-\mathrm{k}, \boldsymbol{\Sigma})$ and $\mathrm{N}=\mathrm{n}_{1}+\cdots+\mathrm{n}_{\mathrm{k}}$. On the other hand, we have

$$
\left|\mathbf{S}+\sum_{\mathrm{i}=1}^{\mathrm{k}} \mathrm{n}_{\mathrm{i}} \boldsymbol{\mu}_{\mathrm{i}} \boldsymbol{\mu}_{\mathrm{i}}^{\prime}\right|=|\mathbf{S}|\left|\mathbf{I}_{\mathrm{p}}+\mathbf{S}^{-1 / 2} \sum_{\mathrm{i}=1}^{\mathrm{k}} \mathrm{n}_{\mathrm{i}} \boldsymbol{\mu}_{\mathrm{i}} \boldsymbol{\mu}_{\mathrm{i}}^{\prime} \mathbf{S}^{-1 / 2}\right| \text {. }
$$

Thus it is enough to minimize the term

$$
\left|\mathbf{I}_{\mathrm{p}}+\mathbf{S}^{-1 / 2} \sum_{\mathrm{i}=1}^{\mathrm{k}} \mathrm{n}_{\mathrm{i}} \boldsymbol{\mu}_{\mathrm{i}} \boldsymbol{\mu}_{\mathrm{i}}^{\prime} \mathbf{S}^{-1 / 2}\right|
$$

Then our problem is to minimize the following term

$$
1+\operatorname{tr}\left(\mathbf{S}^{-1 / 2} \sum_{\mathrm{i}=1}^{\mathrm{k}} \mathrm{n}_{\mathrm{i}} \boldsymbol{\mu}_{\mathrm{i}} \boldsymbol{\mu}_{\mathrm{i}}^{\prime} \mathbf{S}^{-1 / 2}\right)=1+\sum_{\mathrm{i}=1}^{\mathrm{k}} \mathrm{n}_{\mathrm{i}} \boldsymbol{\mu}_{\mathrm{i}} \mathbf{S}^{-1} \boldsymbol{\mu}_{\mathrm{i}}^{\prime},
$$


under the order hypothesis $\boldsymbol{\mu}_{1} \geq \mathbf{0}, \boldsymbol{\mu}_{2} \geq \mathbf{0}, \ldots, \boldsymbol{\mu}_{\mathrm{k}} \geq \mathbf{0}$.

Therefore, for the problem of testing $\mathrm{H}_{0}$ against $\mathrm{H}_{1}$, we get the following test statistic

$$
\mathrm{T}^{\prime}=\sum_{\mathrm{i}=1}^{\mathrm{k}} \mathrm{n}_{\mathrm{i}} \boldsymbol{\mu}_{\mathrm{i}}^{*} \mathbf{S}^{-1} \boldsymbol{\mu}_{\mathrm{i}}^{*^{\prime}}
$$

for when the covariance matrices of populations are unknown but common.

\section{Estimation the critical values and medical application}

In this section, the critical values of the test statistic $T^{\prime}$ are estimated by Monte Carlo simulation method. In this simulation, we generate $n_{1}, n_{2}, \ldots, n_{k}, k=3,4,5$, sets of $p-$ variate normal vectors, $p=3,4,5$, with different sample variance covariance matrices and compute the statistic $T^{\prime}$. This computation is repeated 10000 times to get an estimated upper $\alpha$ point of $T^{\prime}$. We further repeat this process 10 times and compute the average of the 10 estimated upper $\alpha$ point for $\alpha=0.01,0.025,0.05, n_{i}=5,10,15,20,25$, $i=1,2, \ldots, k$, respectively. The estimated critical values are given in Table 1 . Also the critical values of test statistic are estimated when the sample sizes are unequal. The estimated critical values are given in Table 2.

Table 1: Estimated critical values of test statistic when the sample sizes are equal

\begin{tabular}{|c|c|c|c|c|c|c|c|}
\hline & & & & $n_{1}=n_{2}$ & $\cdots=n_{k}$ & & \\
\hline$\alpha$ & $p$ & $k$ & 5 & 10 & 15 & 20 & 25 \\
\hline \multirow[t]{3}{*}{0.01} & 3 & 3 & 2.734 & 2.381 & 1.160 & 0.742 & 0.273 \\
\hline & 4 & 5 & 2.916 & 1.049 & 0.825 & 0.535 & 0.414 \\
\hline & 5 & 4 & 1.250 & 0.635 & 0.341 & 0.251 & 0.123 \\
\hline \multirow[t]{3}{*}{0.025} & 3 & 3 & 1.687 & 1.216 & 0.732 & 0.418 & 0.084 \\
\hline & 4 & 5 & 1.662 & 0.841 & 0.615 & 0.416 & 0.240 \\
\hline & 5 & 4 & 0.631 & 0.452 & 0.243 & 0.142 & 0.046 \\
\hline \multirow[t]{3}{*}{0.05} & 3 & 3 & 1.120 & 0.667 & 0.395 & 0.223 & 0.055 \\
\hline & 4 & 5 & 0.547 & 0.623 & 0.352 & 0.335 & 0.071 \\
\hline & 5 & 4 & 0.346 & 0.381 & 0.187 & 0.065 & 0.026 \\
\hline
\end{tabular}


Table 2: Estimated critical values of test statistic when the sample sizes are unequal

\begin{tabular}{|c|c|c|c|c|c|c|c|c|}
\hline$\alpha$ & $p$ & $k$ & $n_{1}$ & $n_{2}$ & $n_{3}$ & $n_{4}$ & $n_{5}$ & $\begin{array}{l}\text { Critical } \\
\text { value }\end{array}$ \\
\hline \multirow[t]{9}{*}{0.01} & 3 & 3 & 8 & 12 & 21 & 18 & & 4.012 \\
\hline & & & 15 & 10 & 31 & 19 & & 3.209 \\
\hline & & & 16 & 23 & 22 & 20 & & 2.544 \\
\hline & 4 & 5 & 20 & 15 & 25 & 13 & 10 & 2.112 \\
\hline & & & 22 & 21 & 23 & 20 & & 1.730 \\
\hline & & & 23 & 21 & 24 & 20 & & 1.275 \\
\hline & 5 & 4 & 15 & 18 & 26 & 31 & & 1.015 \\
\hline & & & 23 & 28 & 27 & 21 & & 0.883 \\
\hline & & & 26 & 19 & 39 & 25 & & 0.441 \\
\hline \multirow[t]{9}{*}{0.025} & 3 & 3 & 8 & 12 & 21 & 18 & & 3.725 \\
\hline & & & 15 & 10 & 31 & 19 & & 2.850 \\
\hline & & & 16 & 23 & 22 & 20 & & 2.152 \\
\hline & 4 & 5 & 20 & 15 & 25 & 13 & 10 & 2.006 \\
\hline & & & 22 & 21 & 23 & 20 & & 1.429 \\
\hline & & & 23 & 21 & 24 & 20 & & 0.803 \\
\hline & 5 & 4 & 15 & 18 & 26 & 31 & & 0.425 \\
\hline & & & 23 & 28 & 27 & 21 & & 0.081 \\
\hline & & & 26 & 19 & 39 & 25 & & 0.036 \\
\hline \multirow[t]{9}{*}{0.05} & 3 & 3 & 8 & 12 & 21 & 18 & & 3.452 \\
\hline & & & 15 & 10 & 31 & 19 & & 2.840 \\
\hline & & & 16 & 23 & 22 & 20 & & 2.573 \\
\hline & 4 & 5 & 20 & 15 & 25 & 13 & 10 & 1.861 \\
\hline & & & 22 & 21 & 23 & 20 & & 1.200 \\
\hline & & & 23 & 21 & 24 & 20 & & 0.723 \\
\hline & 5 & 4 & 15 & 18 & 26 & 31 & & 0.395 \\
\hline & & & 23 & 28 & 27 & 21 & & 0.074 \\
\hline & & & 26 & 19 & 39 & 25 & & 0.024 \\
\hline
\end{tabular}

\subsection{Real data example (Robertson, et al. 1988)}

To show the obtained results a medical data example is presented. The effect of a drug is investigated on the body of one-day babies for suspected Sepsis disease. An experiment is carried out to evaluate its effect. For this, we consider three factors: Hyporflexia, Hypothermia and Jaundice. According to the medical roles, the normal range of these factors are between 9.0 to 34.0. At the beginning of taking the medication by the doctor, the mean of one-day babies whom had Sepsis disease were zero, but over time, according to observations, the mean of such babies have increased from zero. The experiment was conducted in four different hospitals in four cities. In each hospital, 10 one-day babies have been studied and their three factors have been measured. Let $\boldsymbol{\mu}_{i}=\left(\mu_{1 i}, \mu_{2 i}, \mu_{3 i}\right)^{\prime}$, $i=1,2,3,4$, be the mean of babies whom have Sepsis disease, and the random vectors of mean of one-day babies are distributed with multivariate normal. The results are given in Table 3. For this table, the random vectors are

$$
\mathbf{X}_{1}=(0.2,0,0.3), \mathbf{X}_{2}=(0.2,0.2,0.3), \mathbf{X}_{3}=(0.3,0.4,0.4), \mathbf{X}_{4}=(0.4,0,0.5) .
$$


It is of interest to test whether or not these factors are affected by new drag. Thus, our goal is to test the null hypothesis $H_{0}^{\prime}: \boldsymbol{\mu}_{1}=\mathbf{0}, \boldsymbol{\mu}_{2}=\mathbf{0}, \boldsymbol{\mu}_{3}=\mathbf{0}, \boldsymbol{\mu}_{4}=\mathbf{0}$ against the alternative hypothesis $H_{1}^{\prime}: \boldsymbol{\mu}_{1} \geq \mathbf{0}, \boldsymbol{\mu}_{2} \geq \mathbf{0}, \boldsymbol{\mu}_{3} \geq \mathbf{0}, \boldsymbol{\mu}_{4} \geq \mathbf{0}$, when the covariance matrices are completely unknown.

Table 3: One-day babies for suspected Sepsis disease

\begin{tabular}{crcccccccccc}
\hline & & \multicolumn{10}{c}{ Sepsis disease } \\
\hline Hospital & Factor & 1 & 2 & 3 & 4 & 5 & 6 & 7 & 8 & 9 & 10 \\
\hline 1 & Hyporflexia & 9.23 & 9.65 & 10.43 & 35.40 & 11.29 & 12.53 & 17.82 & 36.41 & 16.90 & 14.15 \\
& Hypothermia & 10.87 & 13.41 & 16.20 & 21.82 & 14.06 & 25.12 & 11.55 & 16.73 & 20.11 & 12.33 \\
& Jaundice & 31.08 & 37.62 & 35.24 & 34.89 & 16.88 & 23.40 & 9.86 & 12.43 & 20.93 & 25.22 \\
2 & Hyporflexia & 32.19 & 10.54 & 12.10 & 16.32 & 25.60 & 38.16 & 11.20 & 22.90 & 37.12 & 13.63 \\
& Hypothermia & 9.85 & 13.56 & 30.04 & 19.52 & 20.04 & 11.81 & 35.63 & 23.40 & 12.46 & 35.72 \\
& Jaundice & 30.24 & 13.61 & 10.87 & 24.51 & 37.10 & 35.31 & 19.07 & 36.12 & 17.55 & 36.40 \\
3 & Hyporflexia & 12.46 & 13.52 & 19.27 & 35.68 & 18.51 & 37.22 & 23.43 & 27.80 & 37.13 & 12.72 \\
& Hypothermia & 36.33 & 24.25 & 16.73 & 36.44 & 35.98 & 13.49 & 12.80 & 36.31 & 15.35 & 27.94 \\
& Jaundice & 13.07 & 29.83 & 35.02 & 34.88 & 24.09 & 14.64 & 36.21 & 35.04 & 27.86 & 17.81 \\
4 & Hyporflexia & 23.60 & 29.07 & 35.81 & 28.60 & 35.22 & 19.81 & 19.07 & 36.33 & 35.30 & 23.61 \\
& Hypothermia & 12.91 & 11.12 & 18.75 & 29.42 & 23.51 & 20.05 & 22.31 & 31.49 & 23.56 & 13.46 \\
& Jaundice & 34.80 & 19.73 & 35.89 & 34.66 & 17.83 & 35.06 & 14.54 & 14.91 & 36.77 & 18.25 \\
\hline
\end{tabular}

The normal range of these factors is between 9.0 to 34.0 .

The data matrix is given by

$$
\mathbf{X}=\left[\begin{array}{llll}
0.2 & 0.2 & 0.3 & 0.4 \\
0 & 0.2 & 0.4 & 0 \\
0.3 & 0.3 & 0.4 & 0.5
\end{array}\right]
$$

After computing the values of vector $\boldsymbol{\mu}_{\mathrm{i}}^{*}$ and matrix $\mathbf{S}^{-1}$, from the data and (8), the value of test statistic $T^{\prime}$ is 2.54 . For $\alpha=0.05$, the critical value is 2.86 . Therefore, the null hypothesis $H_{0}^{\prime}$ is not rejected.

\section{Further Works}

We note that, it would also be an advantage to obtain the critical values of test statistics given in (7) and (8) at different significance levels for some of the multivariate normal distributions. Also the power and $p$-value of these test statistics can be computed using the Monte Carlo simulation. A new problem arises when we suppose that the covariance matrices have an unknown scale factor. In this case, the main problem is estimation of the unknown parameters under the null and alternative hypothesis to obtain the test statistic. I hope to communicate the results of work on these topics later.

\section{Conclusion}

The geometric interpretation of ordered one-sided hypothesis testing presented. We considered tests for the hypothesis that the mean vectors are zero against one-sided alternatives when the observation vectors are independently and identically distributed as normal with known and unknown covariance matrices. This problem is an extension of 
Kudo (1963) and Glimm et al. (2002) to several multivariate normal distributions. The test statistics computed with known and unknown covariance matrices. To evaluate the results, the effect of a drug investigated on the body of one-day babies for suspected Sepsis disease.

\section{Acknowledgment}

The authors are grateful to the referees for their valuable comments and suggestions which led to improvements of this paper.

\section{References}

1. Anderson, T. W. (1984). An Introduction to Multivariate Statistical Analysis, $2^{\text {nd }}$ edition, John Wiley, New York.

2. Bartholomew, D. J. (1959). A test of homogeneity for ordered alternatives, Biometrika, 46, 36-48.

3. Bazyari, A., and Pesarin, F. (2013). Parametric and permutation testing for multivariate monotonic alternatives. Statistics and Computing, 23(5), 639-652.

4. Bazyari, A., and Chinipardaz, R. (2013). Upper bound for p-value of the test of multivariate normal ordered mean vectors against all alternatives. Communications in Statistics-Theory and Methods, 42(10), 1748-1758.

5. Bazyari, A. (2015). Testing order restrictions on several multivariate normal mean vectors, Journal of the Indiana Statistical Association, 53(1 \& 2), 35-62.

6. Bazyari, A. (2017). Bootstrap approach to test the homogeneity of order restricted mean vectors when the covariance matrices are unknown, Communications in Statistics, Computation and Simulation, 46(9), 7194-7209.

7. Bazyari, A. (2018). A general testing for order restriction on mean vectors of multivariate normal populations, DOI: 10.1080/03610918.2018.1508706, Accepted for Publication in Communications in Statistics, Computation and Simulation.

8. Gamage, J. and Mathew, T. (2008). Inference on mean sub-vectors of two multivariate normal populations with unequal covariance matrices, Statistics and Probability Letters, 78, 420-425.

9. Glimm E., Srivastava, M. S. and Lauter, J. (2002). Multivariate tests of normal mean vectors with restricted alternatives, Communications in Statistics, Computation and Simulation, 31(4), 589-604.

10. Imada, T. (2011). Multivariate two sided tests for normal mean vectors based on approximations of likelihood ratio test, Communications in Statistics, Computation and Simulation, 40, 894-906.

11. Kudo, A. (1963). A multivariate analogue of the one-sided test, Biometrika, 50, 403-418.

12. Mardia, K.V., Kent, J. T. and Bibby, J. M. (1979). Multivariate Analysis (Probability and Matematical Statistics), Academic Press. 
13. Perlman, M. D. (1969). One-sided testing problems in multivariate analysis, The Annals of Mathematical Statistics, 40(2), 549-567.

14. Robertson, T. and Wegman, E. T. (1978). Likelihood ratio tests for order restrictions in exponential families, The Annals of Statistics, 6(3), 485-505.

15. Robertson, T., Wright, F. T. and Dykstra, R. L. (1988). Order Restricted Statistical Inference, New York: John Wiley.

16. Sasabuchi, S., Inutsuka, M. and Kulatunga, D. D. S. (1983). A multivariate version of isotonic regression, Biometrika, 70, 465-472.

17. Sasabuchi, S., Tanaka, K. and Takeshi, T. (2003). Testing homogeneity of multivariate normal mean vectors under an order restriction when the covariance matrices are common but unknown, The Annals of Statistics, 31(5), 1517-1536.

18. Silvapulle, M. J. and Sen, P. K. (2005). Constrained Statistical Inference: Inequality, Order, and Shape Restrictions, New York: John Wiley.

19. Sim, S. and Johnson, R. A. (2004). New tests for multivariate ordered alternatives, Communications in Statistics, Theory and Methods, 33(9), 20272039.

20. Wolfe, P. (1959). The simplex method for quadratic programming, Econometrica, 27, 382-98. 